\title{
CAPÍTULO 07: PERCEPÇÕES DE LICENCIANDOS EM CIÊNCIAS BIOLÓGICAS RELACIONADAS À DISCIPLINA BIOQUÍMICA E SUA TRATATIVA NA EDUCAÇÃO BÁSICA E SUPERIOR
}

\author{
CAPÍTULO 07: PERCEPTIONS OF LICENSES IN BIOLOGICAL SCIENCES \\ RELATED TO BIOCHEMICAL DISCIPLINE AND ITS TREATMENT IN BASIC \\ AND UNIVERSITY EDUCATION
}

\section{CHAPTER 07: PERCEPCIONES DE LICENCIAS EN CIENCIAS BIOLÓGICAS RELACIONADAS CON LA DISCIPLINA BIOQUÍMICA Y SU TRATAMIENTO EN EDUCACIÓN BÁSICA Y SUPERIOR}

\author{
José Sérgio Herculano Gomes da Silva ${ }^{1}$; Andressa Raquel Maciel Barros Pontes ${ }^{2}$; Maria Clara de Souza ${ }^{3}$; Victor \\ Manoel Gonçalves dos Santos ${ }^{4}$; Yasmim Alline de Araújo Castro ${ }^{5}$
}

DOI: https://doi.org/10.31692/978-65-88970-05-8.96-109

\begin{abstract}
RESUMO
A disciplina Bioquímica, trabalhada na Educação Básica e Superior, acumula um caráter tradicional e de difícil assimilação pelos discentes, principalmente por tratar de estruturas invisíveis a olho nu e muitas vezes só imagináveis, sendo importante a percepção dos alunos para melhorias na qualidade do ensino-aprendizagem desse conteúdo. Dessa forma, o presente trabalho teve como objetivo relatar e discutir as percepções de licenciandos em Ciências Biológicas de uma Instituição Federal de Ensino Superior relacionada à disciplina Bioquímica, bem como sua tratativa em sala de aula. Para a respectiva participação, os discentes foram informados dos objetivos da pesquisa e concordaram em participar através do preenchimento de um Termo de Consentimento Livre e Esclarecido (TCLE). Posteriormente, responderam um questionário contendo 14 questões relacionadas a aspectos detalhados acerca de suas experiências ao longo da disciplina. Nesse sentido, os resultados apontaram, em sua maioria, uma avaliação negativa aos métodos utilizados na ministração das aulas não só em termos de dificuldades na absorção dos temas propostos, como também na forma como foram desenvolvidos, apontando assim uma falha principalmente na escolha das chamadas metodologias ativas para aplicação do conteúdo. Além disso, foi observada uma preocupação dos discentes em relação à melhor forma de ministrar a disciplina Bioquímica, através de metodologias adequadas, visto que num futuro próximo os estudantes estarão na posição de professores e terão que decidir quais metodologias aplicarão para permitir uma aprendizagem efetiva a seus alunos. Portanto, cabe uma reflexão em busca de uma melhora incessante no desenvolvimento dos temas propostos de uma forma que não comprometa a qualidade da aula, mas que mantenha o interesse dos discentes durante todo o curso, através de um equilíbrio entre a teoria e a prática. Desse modo, a Bioquímica, como disciplina base da graduação em ciências Biológicas, poderá gerar graduandos mais completos e futuros professores comprometidos em repassar seus conteúdos de uma maneira que facilite o processo de ensino-aprendizagem e uma compreensão efetiva dos temas estudados.
\end{abstract}

Palavras-Chave: Formação Docente; Ensino de Bioquímica; Metodologias para o Ensino de Ciências.

\section{RESUMEN}

La disciplina de Bioquímica, trabajada en Educación Básica y Superior, acumula un carácter tradicional y es de difícil asimilación por parte de los estudiantes, principalmente porque se trata de estructuras invisibles a simple vista y muchas veces solo imaginables, siendo importante la percepción de los

\footnotetext{
${ }^{1}$ Licenciatura em Ciências Biológicas, Universidade Federal Rural de Pernambuco, sergio.hercula

${ }^{2}$ Licenciatura em Ciências Biológicas, Universidade Federal Rural de Pernambuco, andressa.pon

${ }^{3}$ Licenciatura em Ciências Biológicas, Universidade Federal Rural de Pernambuco, clara.s

${ }^{4}$ Licenciatura em Ciências Biológicas, Universidade Federal Rural de Pernambuco, vict

${ }^{5}$ Doutoranda em Biologia Celular e Molecular Aplicada, Universidade de Pernambuco; Licenciatura em Ciências Biológicas, Universidade Federal Rural de Pernambuco, yasmim.castro@ufrpe.br
} 
estudiantes para mejoras en la calidad de la enseñanza. aprender ese contenido. Así, el presente estudio tuvo como objetivo informar y discutir las percepciones de estudiantes de licenciatura en Ciencias Biológicas de una Institución Federal de Educación Superior relacionadas con la disciplina de Bioquímica, así como su tratamiento en el aula. Para la respectiva participación, los estudiantes fueron informados de los objetivos de la investigación y acordaron participar completando un Formulario de Consentimiento Libre e Informado (ICF). Posteriormente, respondieron un cuestionario que contenía 14 preguntas relacionadas con aspectos detallados sobre sus experiencias a lo largo de la disciplina. En este sentido, los resultados arrojaron, en la mayoría de los casos, una valoración negativa de los métodos utilizados en la impartición de clases, no solo en cuanto a las dificultades en la absorción de los temas propuestos, sino también en la forma en que fueron desarrollados, señalando así una falla principalmente en la elección de convocatorias. metodologías activas para la aplicación del contenido. Además, hubo preocupación entre los estudiantes por la mejor manera de enseñar la disciplina de Bioquímica, através de metodologías adecuadas, ya que en un futuro cercano los estudiantes estarán en la posición de docentes y tendrán que decidir qué metodologías aplicar para permitir un aprendizaje efectivo. Tus estudiantes. Por tanto, es necesario reflexionar sobre la búsqueda de una mejora continua en el desarrollo de los temas propuestos de forma que no comprometa la calidad de la clase, pero que mantenga el interés de los alumnos a lo largo del curso, mediante un equilibrio entre teoría y práctica. De esta forma, la Bioquímica, como disciplina básica de las carreras de grado en Ciencias Biológicas, puede generar egresados y futuros docentes más completos comprometidos con la transmisión de sus contenidos de manera que facilite el proceso de enseñanza-aprendizaje y una comprensión efectiva de los temas estudiados.

Palabras clave: Formación del profesorado; Enseñanza de la bioquímica; Metodologías para la enseñanza de las ciencias.

\section{ABSTRACT}

The Biochemistry discipline, worked in Basic and Higher Education, accumulates a traditional character and of difficult assimilation by the students, mainly because it deals with structures invisible to the naked eye and often only imaginable, being important the students' perception for improvements in the quality of teaching-learning of that content. Thus, the present study aimed to report and discuss the perceptions of undergraduate Biological Sciences students from a Federal Institution of Higher Education related to the Biochemistry discipline, as well as their treatment in the classroom. For the respective participation, the students were informed of the research objectives and agreed to participate by completing a Free and Informed Consent Form (ICF). Subsequently, they answered a questionnaire containing 14 questions related to detailed aspects about their experiences throughout the discipline. In this sense, the results showed, in most cases, a negative evaluation of the methods used in the teaching of classes, not only in terms of difficulties in absorbing the proposed themes, but also in the way they were developed, thus pointing out a failure mainly in the choice of calls active methodologies for applying the content. In addition, a concern was observed among students regarding the best way to teach the Biochemistry discipline, through appropriate methodologies, since in the near future students will be in the position of teachers and will have to decide which methodologies to apply to allow effective learning to your students. Therefore, it is necessary to reflect on the search for a continuous improvement in the development of the proposed themes in a way that does not compromise the quality of the class, but that maintains the interest of the students throughout the course, through a balance between theory and practice. In this way, Biochemistry, as the basic discipline of undergraduate courses in Biological Sciences, can generate more complete graduates and future teachers committed to passing on its contents in a way that facilitates the teaching-learning process and an effective understanding of the topics studied.

Keywords: Professor Formation; Biochemistry Teaching; Methodologies to Science Teaching.

\section{INTRODUÇÃO}

A disciplina Bioquímica é responsável pela descrição de estruturas moleculares, mecanismos e processos químicos compartilhados pelos organismos e fornece princípios de 
organização da vida em suas diversas formas e princípios (NELSON; COX, 2014). Segundo as orientações complementares aos parâmetros curriculares nacionais (BRASIL, 2006), a partir dos conhecimentos atrelados à Bioquímica e a outras disciplinas, tais como Citologia e Genética, os cidadãos poderão perceber que todas as formas de vida são reconhecidas pela sua organização celular, com origem única.

Tais conhecimentos se mostram fundamentais para um posicionamento no debate contemporâneo sobre as tecnologias de manipulações da vida (BRASIL, 2006). Frente à sua importância, destacamos a necessidade de que a referida disciplina seja trabalhada de forma adequada na Educação Básica, o que depende diretamente da qualidade da formação do docente de Ciências e Biologia para a Bioquímica. Segundo Souza et al. (2020), devido ao grau de abstração associado à Bioquímica, ensinar o conteúdo é muitas vezes uma tarefa complicada para os docentes, principalmente no que se refere às dificuldades de adequação.

Nogara et al. (2018) avaliaram a percepção de discentes de diversos cursos superiores de uma Instituição de Ensino brasileira, e perceberam que em geral os alunos classificam os docentes entre razoável a bom, e que apresentam apenas compreensão razoável da disciplina Bioquímica. Além disso, os autores destacam que a disciplina é oferecida em momento inadequado na grade curricular da maioria dos cursos, e que a mesma só deve ser trabalhada posteriormente às disciplinas Química e Biologia Celular.

Tendo em vista a importância da qualidade do ensino, se faz necessária a avaliação constante de disciplinas inerentes à formação de professores da Educação Básica. Destacamos a Bioquímica como fundamental para a formação de professores de Ciências e Biologia, bem como a discussão das percepções dos atores participantes do processo. Dessa forma, o presente estudo tem como objetivo relatar e discutir as percepções de Licenciandos em Ciências Biológicas de uma Instituição Federal de Ensino Superior relacionada a disciplina Bioquímica, e sua tratativa em sala de aula.

\section{FUNDAMENTAÇÃO TEÓRICA}

A disciplina Bioquímica trabalha estruturas moleculares, os mecanismos e os processos químicos compartilhados por todos os organismos e fornece princípios de organização subjacentes à vida em suas distintas formas e princípios, referidos coletivamente como a lógica molecular da vida (SOUZA et al., 2020). A Bioquímica é uma disciplina básica presente nos cursos de formação da área da saúde, sendo uma das que mais apresenta dificuldade de compreensão entre os alunos, e tal problemática já começa nos primeiros contatos com o 
conteúdo ainda no ensino médio (SCHIMIDT et al., 2014).

Por não serem visíveis a olho nu, entender estes conteúdos têm sido complicado para os estudantes, assim como para os professores que também enfrentam dificuldades no que se refere à adequação da disciplina (SOUZA et al., 2020). Por isso, ensinar a disciplina de Bioquímica é um desafio e, na maioria das vezes, essa disciplina ainda vem sendo ministrada de maneira tradicional, utilizando-se puramente de aulas expositivas, em Datashow, e aulas práticas, complementares ao aprendizado teórico (MATTA; NETO., 2015).

Não é raro encontrar situações em que os estudantes se sentem desinteressados, porque não conseguem perceber a relação entre os conteúdos que estão estudando com vivências relacionadas ao dia-a-dia, sem que o processo de ensino-aprendizagem seja gerador de sentido. Além disso, o conteúdo curricular de Bioquímica é particularmente difícil para o aluno, principalmente por dois motivos: necessidade de conhecimentos científicos prévios sobre propriedades das substâncias presentes no organismo, e concordar que determinadas reações ocorrem, mesmo sem a visualização das interações entre as moléculas e transformações sofridas pelas mesmas, trazendo uma dificuldade de compreensão desses conteúdos trabalhados pelos docentes (SCHIMIDT et al., 2014).

No ensino das Ciências Naturais, no qual se faz necessário o uso de diferentes metodologias de ensino-aprendizagem, a experimentação é obviamente uma estratégia essencial que pode ajudar na melhor compreensão dos fenômenos estudados, como os materiais multimidiáticos para mostrar a dinâmica das macromoléculas biológicas como parte essencial para sua função (DIAS et al., 2013).

\section{METODOLOGIA}

Para alcance dos objetivos propostos por esse trabalho, a pesquisa teve como público alvo licenciandos em ciências biológicas de uma Instituição Federal de Ensino Superior de Pernambuco. Para participação na pesquisa, foi necessário que os discentes já tivessem cursado a disciplina Bioquímica na referida Instituição de Ensino, e dessa forma pudessem expor suas percepções relacionadas à Bioquímica.

O contato com os convidados da pesquisa se deu por meio do aplicativo digital WhatsApp, respeitando as orientações da Organização Mundial de Saúde (OMS) face à pandemia de COVID-19, garantindo a segurança de participantes e pesquisadores. Logo após o contato inicial, foram explanados os objetivos da pesquisa e realizado o convite à participação por meio da concordância com um Termo de Consentimento Livre e Esclarecido (TCLE), que 
garantia aos participantes anonimato e retirada de seus dados da pesquisa quando desejado, bem como a publicação dos dados obtidos em meios científicos.

Para a coleta de dados, utilizou-se um questionário semiestruturado contendo 14 questões, sendo três que buscavam conhecer o perfil dos entrevistados, e 11 contemplando suas percepções e experiências relacionadas à disciplina Bioquímica. Segundo Chaer et al. (2011), o questionário se mostra como uma excelente ferramenta metodológica, principalmente por garantir o anonimato dos participantes e ser de fácil manejo na padronização dos dados.

Visando garantir a segurança de participantes e pesquisadores, o envio dos formulários se deu via WhatsApp, utilizando-se de um link gerado pela plataforma Google Forms, que permite a coleta de informações de forma fácil e eficiente. Após as respostas dos participantes, os dados foram analisados e transformados em textos e gráficos para melhor visualização, organização e entendimento.

\section{RESULTADOS E DISCUSSÃO}

A pesquisa foi composta por 40 licenciandos em ciências biológicas de uma Instituição Federal de Ensino Superior de Pernambuco. Dentre os participantes do presente estudo, 25 (62,5 $\%)$ eram do sexo feminino e $15(37,3 \%)$ do sexo masculino. A faixa etária mais predominante era entre 18 a 25 anos, correspondendo a $90 \%$ dos entrevistados (36 indivíduos). No que diz respeito ao período letivo, $72,5 \%$ dos entrevistados estavam no terceiro período, tendo cursando Bioquímica recentemente em duas disciplinas dos períodos anteriores.

Na primeira pergunta, os participantes foram questionados acerca do primeiro contato que tiveram com os conteúdos relacionados à disciplina Bioquímica, como pode ser observado na figura 1. Dos 40 alunos entrevistados, $24(60 \%)$ tiveram o primeiro contato no ensino médio, enquanto 10 participantes $(25 \%)$ tiveram no ensino fundamental. Tal resultado é semelhante ao de uma pesquisa realizada para saber os conhecimentos prévios de Bioquímica de alunos de vários cursos da área da saúde. A grande maioria afirmou que a fonte de obtenção destes conhecimentos foi o ensino médio (50,6\%); além disso, 27,6\% não responderam, seguidos por cursinho $(16,1 \%)$, em casa $(4,6 \%)$ e em outra universidade $(2,3 \%)$ (ZENI, 2010).

Entretanto, podemos observar que, no presente estudo, seis indivíduos (15\%) tiveram contato inicial com a disciplina Bioquímica apenas no ensino superior. Tal percentual demonstra que, para esses alunos, a aprendizagem da Bioquímica é um desafio, sendo necessário muito empenho dos mesmos e dos professores, a fim de superar tal dificuldade. Segundo Zeni (2010), considerando a importância de se ter conhecimentos prévios para um 
melhor entendimento da Bioquímica, é preocupante perceber que muitos alunos não possuem tais conhecimentos, o que dificulta a aprendizagem e transformação do conhecimento em algo palpável e entendível e, consequentemente, dificultando a formação dos discentes no universo da Bioquímica.

Figura 1. Primeiro contato dos alunos com os conteúdos de Bioquímica no ambiente escolar.

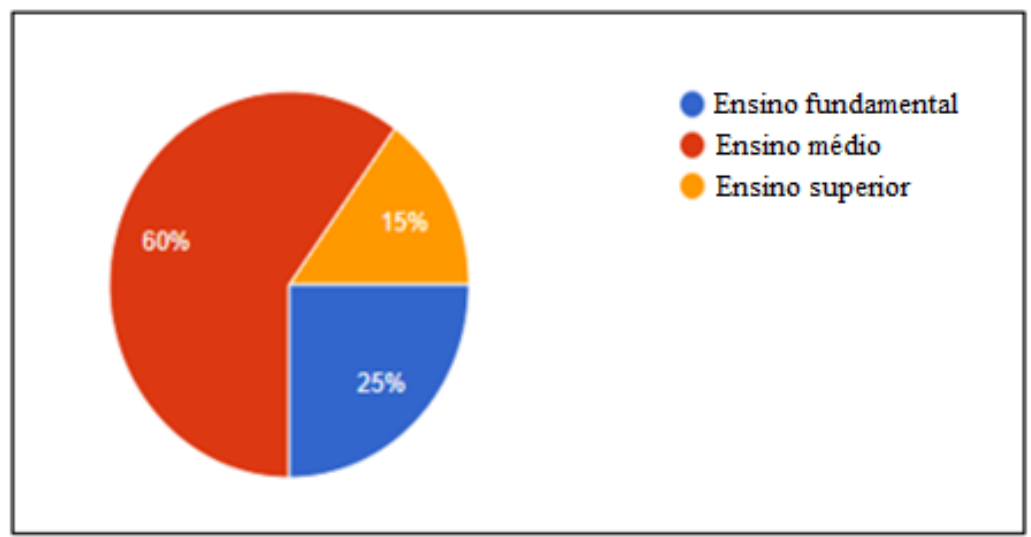

Fonte: Própria (2020).

$\mathrm{Na}$ segunda questão, foi perguntado se os alunos acreditavam que os conteúdos de Bioquímica foram trabalhados de forma adequada na educação básica dos mesmos, conforme observado na figura 2. Dos indivíduos entrevistados, a maioria (22 alunos - $55 \%$ ) consideravam que os conteúdos de Bioquímica na educação básica foram trabalhados com bastante dificuldade; 14 participantes (35\%), por sua vez, responderam que os conteúdos foram trabalhados de modo parcial e, por isso, necessitaram de algumas revisões para relembrar determinados assuntos. Dos entrevistados, apenas quatro participantes $(10 \%)$ responderam que tiveram uma ótima base durante a educação básica. Dessa forma, a maioria dos alunos chegou ao ensino superior com um déficit nos conteúdos de Bioquímica, o que influenciou negativamente o aprendizado dos mesmos.

Em uma pesquisa realizada para entender as dificuldades de aprendizagem em relação à Bioquímica em um curso superior da área da saúde, 84 a 96\% dos respondentes (n=47 a 50 alunos, $100 \%$ dos matriculados), concordaram ou concordaram fortemente que a Bioquímica é importante para sua profissão. Entretanto, para os mesmos, a principal dificuldade encontrada para a aprendizagem de Bioquímica foi a falta de uma base em Química, correspondendo entre 77 a $95 \%$ dos estudantes entrevistados (SCATIGNOL; TORRES, 2016). A dificuldade em química dos alunos de bioquímica pode ser explicada pelo contato ainda prematuro com essa área, visto que a disciplina é ofertada geralmente no primeiro ano dos cursos e, além disso, tal contato está associado a um ensino médio deficiente (ZENI, 2010; ANDRADE et al., 2017). 
Figura 2. Percepção dos alunos sobre a aprendizagem de Bioquímica na educação básica.

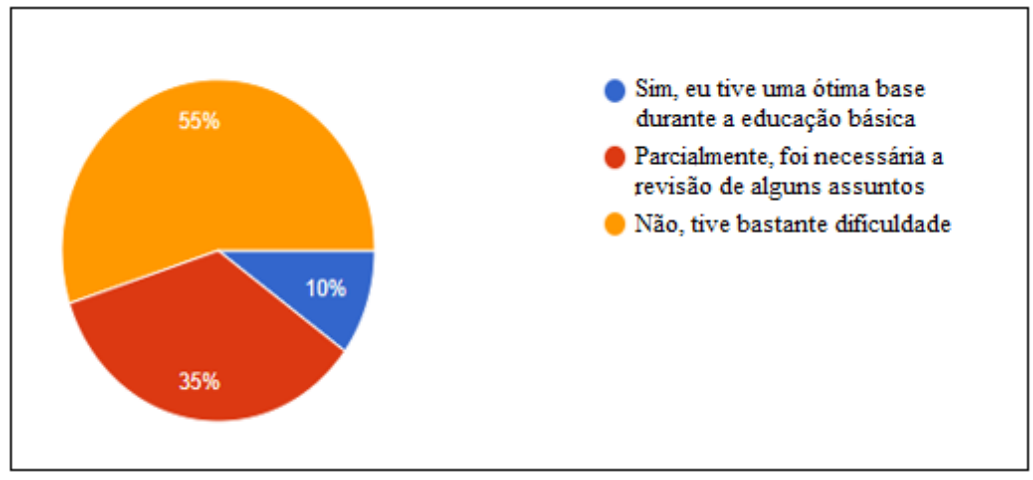

Fonte: Própria (2020).

$\mathrm{Na}$ terceira questão, cujo tema era quais as principais metodologias utilizadas pelos professores de Bioquímica no ensino superior, verificamos que, na percepção da maioria dos participantes (22, correspondendo a $55 \%$ dos entrevistados), os docentes fizeram uso predominantemente de metodologias ativas. Além disso, 13 participantes (32,5\%) afirmaram que os docentes fizeram uso de um modelo que equilibrou teoria e prática, e três participantes $(7,5 \%)$ afirmaram que os docentes ministraram aulas predominantemente teóricas, baseandose no uso do quadro, slides e leituras. Para Person e colaboradores (2020), um dos grandes desafios do ensino de Bioquímica é desenvolver nos alunos a capacidade de verem sentido aos temas abordados, a fim de criarem possibilidades de aprendizagem e estimulação do pensamento crítico.

Em uma pesquisa, cujo objetivo foi investigar os motivos do baixo rendimento de alunos do curso de Nutrição de uma Instituição de Ensino Superior da rede particular, os pesquisadores observaram que os discentes possuíam grande dificuldade com conceitos químicos e com a intepretação das representações simbólicas da Bioquímica (SCATIGNOL et al., 2016). Dessa forma, o uso de metodologias ativas de forma predominante pode prejudicar o aprendizado, tendo em vista que é necessária uma preparação com conceitos e pontos-chave prévios, a fim de se permitir o entendimento da representação que o professor escolher, tais como desenhos, maquetes, esquemas e outras metodologias ativas.

Conforme Monteiro e colaboradores (2006), a aprendizagem de Bioquímica depende de símbolos e das representações externas, e as ferramentas de visualização dão suporte ao entendimento e à pesquisa em biociências celulares e moleculares. Dessa forma, tais representações externas permitem que os professores representem a Bioquímica através de modelos e metodologias ativas, a fim de facilitar a visualização e integração dos conceitos estudados. Porém, tendo em vista que os discentes podem ter dificuldade para entenderem as representações externas, é fundamental que os professores busquem sempre a melhor maneira 
de ensinar e interpretar tais representações, a fim de permitirem uma aprendizagem efetiva (SCHÖNBORN et al., 2006; SCATIGNOL et al., 2016).

Figura 3. Metodologias utilizadas pelos professores de Bioquímica no ensino superior.

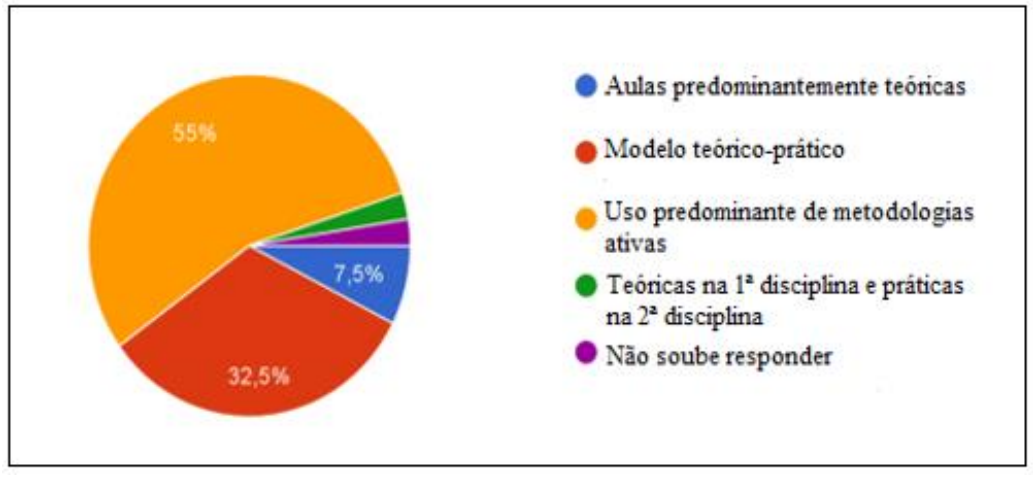

Fonte: Própria (2020).

Na quarta questão, apurou-se o nível de dificuldade que o aluno associa ao aprendizado de Bioquímica, conforme demonstrado na figura 4. Constatamos que 22 participantes (a maioria) destacaram um grau de dificuldade mediana $(57,5 \%)$ e 10 participantes $(25 \%)$ consideram difícil o aprendizado da disciplina de Bioquímica. Outros cinco participantes (12,5 $\%)$ afirmaram que possuem pouca dificuldade e dois participantes (5\%) associaram o aprendizado da Bioquímica a um nível muito difícil.

Para analisar de uma forma geral o grau de dificuldade de aprendizado dos alunos de vários cursos da saúde na disciplina de Bioquímica, pesquisadores agruparam os escores em quatro graus: nenhum, baixo, médio e alto. De forma semelhante ao nosso estudo, constataram que a maioria dos discentes possuía um grau de dificuldade média $(44,5 \%)$ ou alta (42\%) (ZENI, 2010). Segundo Pinheiro e colaboradores (2009), apesar dos esforços para apresentar a Bioquímica de uma forma estruturada e coerente, ela é vista pelos estudantes como uma disciplina complexa e de difícil assimilação, tendo em vista a complexidade das estruturas químicas e vias metabólicas, por exemplo.

Figura 4. Nível de dificuldade associado ao aprendizado de Bioquímica.

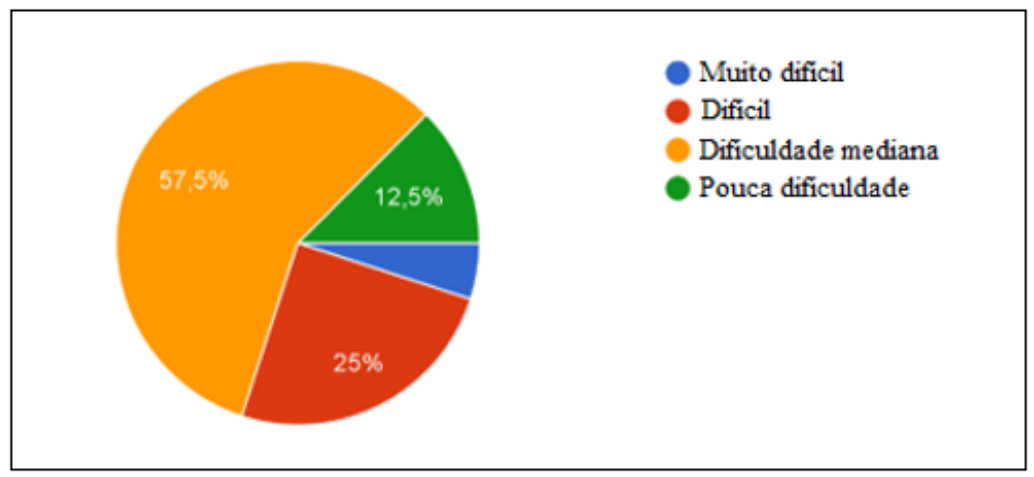

Fonte: Própria (2020). 
$\mathrm{Na}$ quinta questão, os discentes explanaram as principais dificuldades associadas ao aprendizado da disciplina Bioquímica no ensino superior. Como resultado, 21 participantes $(52,5 \%)$ destacaram que o principal motivo foi a metodologia escolhida pelo docente da disciplina, nove participantes $(22,5 \%)$ afirmaram que o principal motivo foi a utilização de termos científicos de difícil compreensão; outros motivos marcados pelos participantes foram a falta de dedicação à disciplina (4 discentes - $10 \%$ ) e outros quatro participantes $(10 \%)$ afirmaram não ter dificuldade no aprendizado de Bioquímica (Figura 5).

Em uma pesquisa realizada para investigar as principais dificuldades de aprendizado em Bioquímica de estudantes de diversos cursos da área da saúde de uma Instituição Federal de Ensino, os principais motivos citados pelos alunos foram o excesso de conceitos envolvidos no estudo da Bioquímica (54\%), seguido do fato de eles considerarem o volume de informação muito grande a ser abordado em pouco tempo $(48,2 \%)$ e a presença de outras disciplinas complexas na grade curricular de cada curso (39,4\%) (ANDRADE et al., 2017).

A Bioquímica é uma disciplina considerada de difícil compreensão, tendo em vista que exige um nível elevado de abstração ao tratar de fenômenos a nível molecular (PERSON et al., 2020). Além disto, na maioria das vezes as percepções e avaliação sobre a disciplina de Bioquímica por estudantes de graduação são bastante negativos, o que demonstra a necessidade de se repensar o modo de ensino da disciplina, de uma maneira que permita um aprendizado eficaz e, ao mesmo tempo possível, com aprimoramento dos conhecimentos básicos (NOGARA et al., 2018).

Figura 5. Principais dificuldades associadas ao aprendizado de Bioquímica no ensino superior.

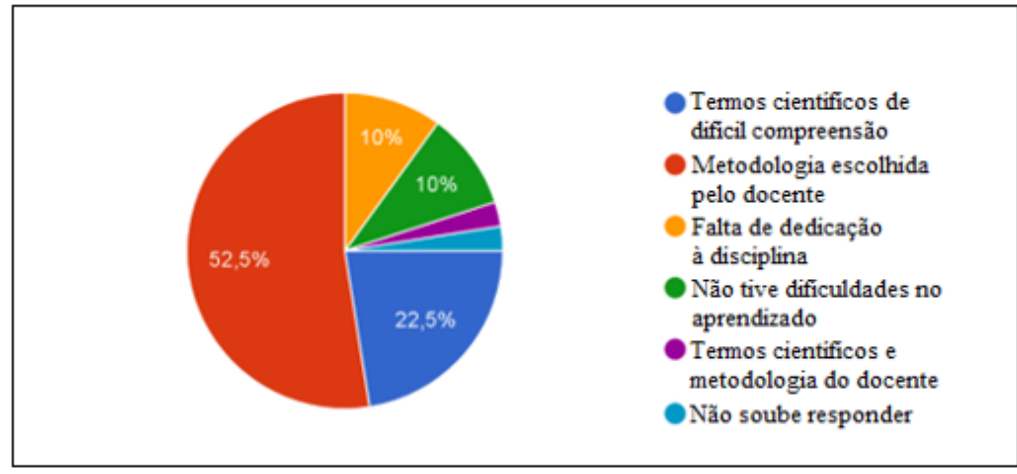

Fonte: Própria (2020).

$\mathrm{Na}$ sexta questão indagou-se acerca da necessidade ou não de se interdisciplinaridade no ensino da Bioquímica, visto que é uma disciplina que embasa outras, como a Fisiologia. A grande maioria dos discentes $(30-75 \%)$ enfatizou a importância do ensino interdisciplinar da Bioquímica; outros nove alunos $(22,5 \%)$, por sua vez, consideram a interdisciplinaridade necessária apenas em algumas aulas. Em contrapartida, apenas um aluno 
$(2,5 \%)$ não encontrou validade nesse tipo de abordagem (Figura 6).

É sabido que a Bioquímica representa uma área interdisciplinar por si só, tendo em vista que uma vez que possui como base as Ciências Biológicas e Químicas. Dessa forma, constituise como um eixo temático deveras amplo e promissor para o desenvolvimento de abordagens disciplinares (JUNIOR, 2007). Para Beckhauser et al. (2006), a integração horizontal, definida pela inter-relação entre as disciplinas cursadas no mesmo semestre, é apontada como um dos motivos da segmentação dos processos de aprendizagem e impedimento da interdisciplinaridade, tendo em vista que o estudante não consegue aproximar o conhecimento das disciplinas básicas com o conhecimento das disciplinas específicas. Isso leva à fragmentação do conhecimento e consequentemente não haverá relação entre o que foi aprendido e possíveis aplicações do conhecimento na formação profissional do educando.

Figura 6. Percepção dos discentes acerca da aplicação da interdisciplinaridade no ensino da Bioquímica.

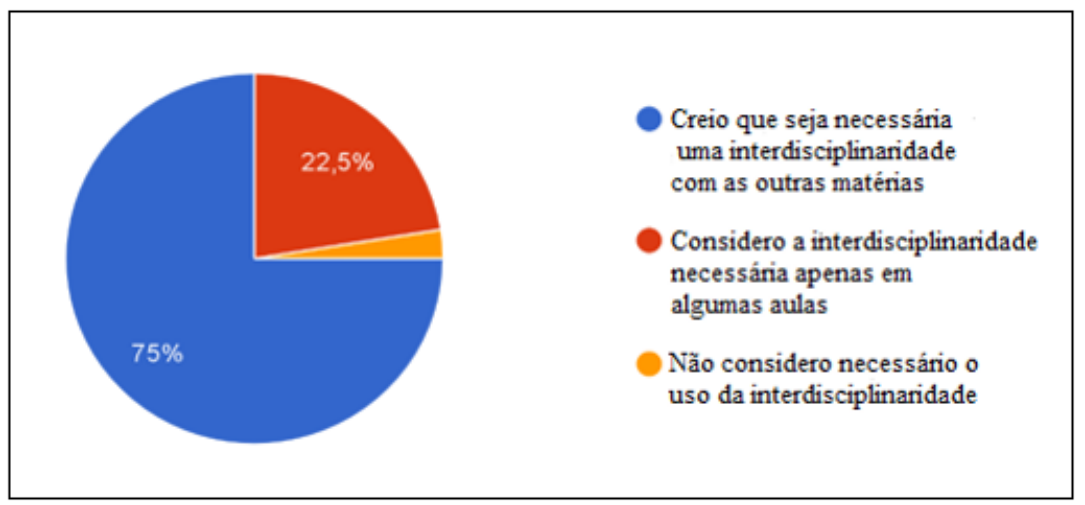

Fonte: Própria (2020).

Na questão sete, cujo objetivo era obter o grau de satisfação dos estudantes em relação à aprendizagem de Bioquímica no ensino superior, a maioria dos alunos afirmou regular (15 discentes $-37,5 \%$ ), seguido dos escores bom (14 discentes $-35 \%$ ), ótimo (4 alunos $-10 \%$ ), ruim (4 alunos $-10 \%$ ) e péssimo (3 alunos - 7,5\%) (Figura 7).

Figura 7. Grau de satisfação dos discentes em relação à aprendizagem de Bioquímica no ensino superior.

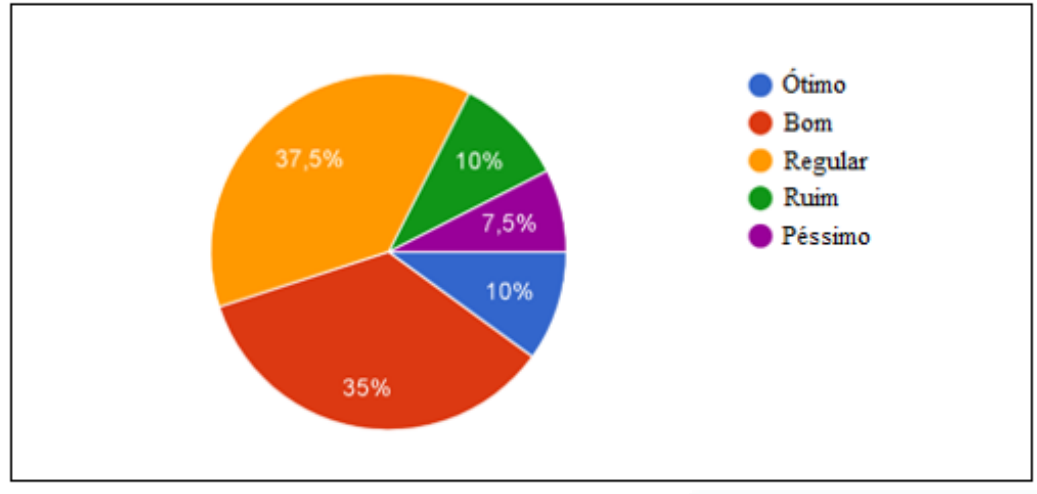

Fonte: Própria (2020).

$\mathrm{Na}$ oitava questão, foi perguntado se a experiência metodológica nas aulas de 
bioquímica obtida por esses estudantes seria replicada por eles no futuro como professores (Figura 8). Nesse sentido, 29 pessoas $(72,5 \%)$ disseram que eles optariam pela mesma metodologia que tiveram em suas aulas de bioquímica da graduação, porém apenas parcialmente. Enquanto isso, 6 pessoas (15\%) afirmaram que buscariam outros métodos e cinco pessoas $(12,5 \%)$ confirmaram que tentariam ao máximo replicar a mesma metodologia que lhes foi passada.

Figura 9. Representação das respostas dos participantes sobre a possível replicação das metodologias para o ensino de Bioquímica, utilizadas em sua graduação como docentes da disciplina

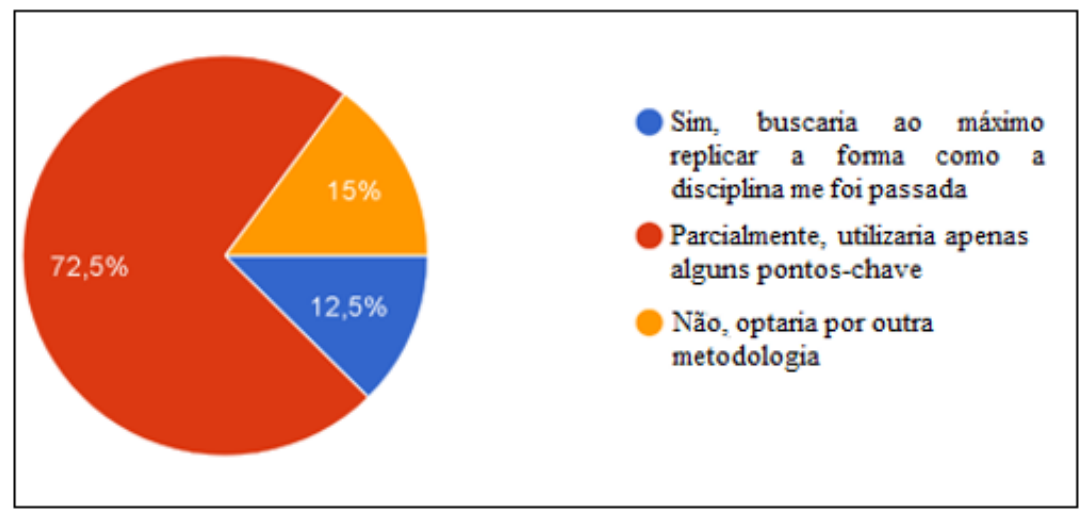

Fonte: Própria (2020).

A décima primeira questão indagou os entrevistados acerca das possíveis sugestões que eles dariam para melhorar o ensino aprendizado da disciplina de bioquímica no curso de Ciências Biológicas. Nessa perspectiva, ao analisar as respostas dos estudantes, nota-se um consenso da maioria dos alunos de como deve ser feita a mudança para uma verdadeira melhoria. Para eles, é necessária uma ampla e reforçada abordagem teórica, somado a isso uma ligação com o campo prático, para que assim o aprendizado seja mais efetivo.

Entretanto, sob outro ângulo um pensamento divergente se ergue ampliando a discussão, certo estudante abordou em sua resposta que a melhoria viria por intermédio de uma aprendizagem que tangenciasse o cotidiano do aluno, além de trazer de maneira lúdica o conhecimento. Conforme tal discente, “o uso moderado de atividades lúdicas que possibilitem o entendimento de determinados processos bioquímicos de difícil visualização pode se tornar uma grande ajuda durante o decorrer da disciplina. Além disso, é uma ótima ideia trazer assuntos do cotidiano, para que a partir disso surjam assuntos específicos da Bioquímica."

\section{CONCLUSÕES}

A partir de uma análise de todos os fatores que compuseram a presente pesquisa e o compilado de discussões geradas a partir dela, algumas considerações podem ser inferidas. 
Nesse viés, observa-se que há a necessidade de uma maior cautela do docente ao propor o estímulo de seus alunos na busca por respostas acerca das problemáticas pautadas em sala de aula. Cabe a ele fornece aos alunos situações que possam relacionar o conteúdo ministrado ao cotidiano diário e/ou técnico, como também se atentar à forma utilizada para compartilhar esses conhecimentos de modo a despertar o interesse da grande maioria de seus discentes.

Propõe-se, à vista disso, um planejamento minucioso e detalhista e a inserção de alternativas de estudo diferenciadas, uma vez que as dificuldades, sejam elas provindas do Ensino Médio ou de semestres anteriores, interferem decisivamente no andamento do curso. Portanto, é de caráter vital um diagnóstico precoce, para que os alunos tenham a oportunidade de construir um conhecimento consistente a ser utilizado de forma capacitada e consistente.

Torna-se evidente afirmar que o aprendizado dos discentes em questão foi impactado com a apresentação de uma abordagem com predominância de metodologias ativas aplicadas pelo docente, sendo necessário um equilíbrio entre o conteúdo teórico, a fim de que o estudante entre em contato com conceitos iniciais, e o conteúdo prático/com metodologias ativas, no qual o discente conseguirá visualizar de modo mais palpável as estruturas e vias metabólicas, por exemplo; além disso, faz-se necessária uma abordagem interdisciplinar, levando em consideração a importância da disciplina Bioquímica no entendimento de outras disciplinas. A partir disso, a dúvida que se estabelece é qual seria a melhor forma de se ministrar os conteúdos da Disciplina Bioquímica; entretanto, debater tais reivindicações abre espaço para um progresso comunicativo, para uma busca pela amenização dos problemas levantados ou ainda para a resolução.

Além disso, percebe-se que, ao estabelecer o contato com essas metodologias, há a identificação de alguns estímulos e/ou pontos-chaves que levam os alunos à reflexão acerca do que está sendo utilizado no momento das aulas e ainda se o modo como isso está sendo feito está promovendo resultados ou positivos ou não. Nessa perspectiva, o debate acerca dessa situação é deveras importante na formação do aluno, tendo em vista que, através do pensamento crítico, o mesmo está formando conceitos e estratégias para ministrar a disciplina quando for professor e, consequentemente, observando os pontos positivos e negativos da experiência com a disciplina Bioquímica, e tirando o melhor de tal experiência para, futuramente, repassar a seus alunos.

\section{REFERÊNCIAS}

ANDRADE, R.S.B.; SILVA, A.F.S.; ZIERER, M.S. Avaliação das dificuldades de aprendizado em Bioquímica dos discentes da Universidade Federal do Piauí. Revista de 
Ensino de Bioquímica, vol 15, nº 1, p.24-39, 2017.

BECKHAUSER, P. F.; ALMEIDA, E. M.; ZENI , A. L. B. O universo discente e o ensino de bioquímica. Revista de Ensino de Bioquímica, vol 4, nº. 2, p.16-22, 2006.

BRASIL, Orientações Complementares aos Parâmetros Curriculares Nacionais (PCN+). Ciências da Natureza e Matemática e suas tecnologias. Brasília: MEC, 2006.

CHAER, G.; DINIZ, R. R. P.; RIBEIRO, E. A. A técnica do questionário na pesquisa educacional. Revista Evidência, vol. 7, nº. 7, p.251-266, 2011.

DIAS, G.; et al. Desenvolvimento de Ferramentas Multimidiáticas para o Ensino de Bioquímica. Revista PRÁXIS, vol 5, nº 9, p.25-30, 2013.

FRAGELLI, R. R. Trezentos: Aprendizagem Ativa e Colaborativa como uma Alternativa ao Problema de Ansiedade em Provas. Revista Eletrônica Gestão e Saúde, vol 6, nº 9, p. 860$872,2015$.

JÚNIOR, W. E. F. Bioquímica no ensino médio?! (de)limitações a partir da análise de alguns livros didáticos de química. Ciência e Ensino, vol 1, nº. 2, 2007.

MATTA, L. D. M.; NETO, L. S. Propostas de Projetos Voltados para o Ensino Básico, Desenvolvidos por Estudantes de Licenciatura. Ensino de Bioquímica e Formação de Docente, vol 38, nº. 3, p.224-229, 2016.

MONTEIRO B. S.; et al. Metodologia de desenvolvimento de objetos de aprendizagem com foco na aprendizagem significativa. Simpósio Brasileiro de Informática na Educação, vol 17, p. 388-397, 2006.

NELSON, D. L.; COX, M. M. Lehninger - Princípios de Bioquímica. 6ed. São Paulo: Sarvier, 2014.

NOGARA, P. A.; et al. Percepção de estudantes de graduação de uma Universidade brasileira sobre a disciplina de Bioquímica. Revista de ensino de Bioquímica, vol 16, nº 1, p. 5-24, 2018.

PERSON, V. A; ROCHA, J. B. T. Inter-relação entre metodologias didáticas, motivos e aprendizagem em Bioquímica. Revista Insignare Scientia, vol. 3, nº. 2 , p.101-108, 2020.

PINHEIRO, T. D. L.; et al. Ensino de Bioquímica para acadêmicos de Fisioterapia: visão e avaliação do discente. Revista de Ensino de Bioquímica, vol 7 , nº 1, p.25-35, 2009. PORTUGAL, L. R.; OLIVEIRA, G. O. Método Trezentos como Estratégia de Ensinoaprendizagem na Disciplina de Bioquímica do Curso de Educação Física. Congresso de Inovação e Metodologias no Ensino Superior, nº 4, 2019.

SCATIGNOL, A. C.; TORRES, B. B. Diagnósticos e intervenções no Ensino de Bioquímica. Journal of Biochemistry Education, vol 24, nº.1, p. 29-51, 2016.

SCHIMIDT, D. B.; et al. Mapas Conceituais no Ensino da Bioquímica, uma Integração entre os Conceitos Científicos. Revista de Ensino de Bioquímica, vol 12, nº. 2, p. 7-23, 2014. 
SCHONBORN, K. J.; ANDERSON, T. R. The importance of Visual Literacy in the Education of Biochemists. Biochemestry and Molecular Biology Education, vol 34, $\mathrm{n}^{\circ} .2$, p. 94-102, 2006.

SOUZA, K. M. S.; et al. Elaboração de modelos moleculares reutilizando materiais para o ensino da Bioquímica. Cadernos de Educação Básica, vol 5, nº. 2, p. 1-17, 2020.

ZENI, A. L. B. Conhecimento prévio para a disciplina de Bioquímica em cursos da área da saúde da Universidade Regional de Blumenau-SC. Revista Brasileira de Ensino de

Bioquímica e Biologia Celular, vol 1, nº. 1, p. B1-B13, 2010. 